\title{
Hypogonadotropic hypogonadism in women: treatment options
}

\author{
Yona Greenman
}

Institute of Endocrinology, Metabolism and Hypertension, Tel Aviv Sourasky Medical Center

Tel Aviv University, Israel

\author{
Address for correspondence: \\ Yona Greenman \\ Institute of Endocrinology \\ Metabolism and Hypertension \\ Tel Aviv Sourasky Medical Center \\ Tel Aviv University \\ 6 Weizmann Street, Tel Aviv, Israel \\ E-mail: yonagr@tlvmc.gov.il
}

\section{Summary}

Treatment of female hypogonadotropic hypogonadism is geared to mimic normal physiology, allowing for age appropriate development, while preventing complications secondary to sex hormone deprivation. In this paper we review data concerning efficacy and safety of sex hormone replacement therapy at the various stages of female reproduction, taking into account safety profiles of different types of estrogen and progesterone formulations, and their interactions with other components of the pituitary substitutive therapy.

KEY WORDS: hypogonadism; hypopituitarism; estrogen; hormone replacement.

\section{Introduction}

As with other deficiencies in hypothalamo-pituitary-target organ axes, treatment of female hypogonadotropic hypogonadism is geared to mimic normal physiology, allowing for age appropriate development, while preventing complications secondary to sex hormone deprivation. That we do not fully achieve this goal is suggested by reports of excess mortality in patients with hypopituitarism (1), which is more pronounced in women than in men (2).

In children, sex steroid hormone replacement is given gradually to simulate normal pubertal development. In adulthood, issues of fertility including induction of ovulation, normal sexual functioning as well as achievement and maintenance of bone health should be addressed. In this paper we will review data concerning efficacy and safety of sex hormone replacement therapy at the various stages of female reproduction, taking into account safety profiles of different types of estrogen and progesterone formulations, and their interactions with other components of the pituitary substitutive therapy.

\section{Puberty and adolescence}

The goal of therapy is to simulate normal puberty, including the development of secondary sex characteristics, uterine and endometrial maturation and bone mass accretion, while fulfilling the genetic potential for height and allowing for psychosocial maturation at an appropriate age. Unfortunately, evidence-based information regarding the optimal treatment schedule for children with hypogonadotropic hypogonadism is sparse, and their clinical management relies on data obtained mainly from studies in girls with Turner's syndrome (3). Regarding the timing of pubertal induction, delaying estrogen therapy to optimize height potential is no longer recommended, more emphasis being given to age appropriate pubertal maturation (4). The initial estrogen dose should be one tenth to one eight of the adult replacement, followed by a gradual dose increase over a period of 2-4 years (4). Progestins should be added at least two years after estrogen initiation, or if breakthrough bleeding occurs.

Oral estrogens, mostly conjugated estrogens in the US and ethinyl estradiol or micronized estradiol in Europe have been the predominant preparations used by pediatric endocrinologists (3), although there seem to be a trend to move to transdermal preparations in the last decade. In the guidelines formulated by the Turner Syndrome Study Group (4) there is no specific recommendation as to the type of estrogen formulation to be used, and dose equivalence is provided for conjugated equine estrogens, ethynil estradiol and micronized estradiol, but it is suggested that treatment regimens using injectable or transdermal estradiol may be more physiological.

Oral estrogen preparations may potentially have deleterious effects on maximal height attainment, as they undergo first-pass metabolism in the liver leading to reduced IGF-1 generation, an effect not seen with physiological doses of transdermal/parenteral estradiol preparations (5). This notion is supported by results of a small study in which girls treated with very small doses of parenteral depot estradiol from the age of 12 achieved significantly more linear growth in comparison with those treated with conjugated estrogens started at a similar age (6). Additional evidence comes from 
a prospective French registry study in which transdermal estradiol treatment was independently associated with improved adult height (7).

Evidence pointing to a possible superiority of the transdermal route in attaining other endpoints of pubertal development has been accumulating. A recent study on estrogen replacement in pre-pubertal girls with Turner syndrome showed that transdermal estradiol, as compared with oral conjugated estrogens, resulted in greater accrual of spine bone mineral content and density, as well as greater increases in uterine length and volume (8). The importance of achieving normal uterine development cannot be overemphasized as it may influence response rates to subsequent fertility treatments. There seems to be a dissociation between breast development and uterine growth in certain regiments of pubertal induction. For example, only $50 \%$ of girls with Turner syndrome with complete secondary sexual development had a mature size and shaped uterus when treated with ethinyl estradiol (9). Similarly, patients with hypopituitarism or hypogonadotropic hypogonadism treated with a standard ethinyl estradiol regimen for induction of puberty were also found to have significantly smaller uterus length and uterus cross-sectional area in comparison to controls (10). In contrast, treatment with increasing doses of estradiol gel resulted in normal uterine development in all patients (11). Consideration should also be given to the dose of estradiol, as a positive correlation has been found between postmenarcheal estrogen dose and uterine length (12). In view of the accumulating evidence in favor of transdermal estradiol for the purpose of puberty induction, its use has significantly increased, not only in patients with primary ovarian failure but also in those with hypogonadotropic hypogonadism (13).

\section{Fertility}

With the advent of ovulation induction with gonadotropins more than five decades ago, women with hypopituitarism should expect near normal fecundity. In contrast to women with isolated hypogonadotropic hypogonadism who were reported to have fertility treatment outcomes similar to that in the general population (14), this was not the case in women with various degrees of hypopituitarism. In a series of 19 patients, pregnancy was achieved in only $47 \%$, with a live birth rate of $6.7 \%$ per cycle (15). Although GH administration in the context of fertility treatment has been shown to be beneficial in hypopituitary patients, pregnancy rates were not affected by the use of GH in this series. Another small case study reported poor pregnancy outcomes in hypopituitary patients: live birth rate was $61 \%$, with $50 \%$ of the babies being small for gestational age or with birth weights below the $10^{\text {th }}$ centile (16). A review of 31 published cases of pregnancies in women with hypopituitarism found similarly increased rates of babies small for gestational age in comparison with pregnancy controls (38 vs $12.7 \%$ ), and a high incidence of transverse lie (15 vs $2.1 \%$ ) (17). It has been hypothesized that uterine size may be a determinant of poor uterine function, and that poor preparation of the uterus prior to conception may have contributed to the poor pregnancy outcomes, emphasizing the need for treatment optimization.

\section{Adulthood}

Most of the knowledge concerning the effects of hormone replacement therapy (HRT) originates from large studies performed in post-menopausal women. The expectations that HRT therapy would reduce cardiovascular disease, as has been suggested from epidemiological studies and from the established beneficial effects of estrogens on the vasculature and lipid profile (18), were shattered by the results of the Women's Health Initiative studies (WHI) (19). As it has been widely publicized, treatment not only failed to prevent vascular outcomes, but was associated with an increased risk for stroke and ischemic heart disease. As a consequence, the use of HRT has substantially decreased in all age groups, despite the fact that the average age of participants in the WHI was 63 years. According to data from the National Health and Nutrition Examination Survery (NHANES), $2.5 \%$ of women aged 40-49 reported current use of oral postmenopausal hormones in the period or 2009-2010, compared to $12.3 \%$ during 1999-2000 (20). Extrapolation of results obtained from treatment of women in late menopause to young hypogonadal women may be misleading and much caution should be exercised (21). It cannot be assumed that estrogen depletion and replacement thereof would have similar consequences in young women, whose endogenous estrogen levels should be significantly higher than those obtained with standard HRT, and in older women in whom a low estrogen milieu is physiologic. Furthermore, morbidity may vary according to the age of onset of estrogen deficiency in the young and its etiology (hypogonadotropic hypogonadism vs primary hypogonadism due to Turner's syndrome, primary ovarian insufficiency, bilateral oophorectomy or following cancer therapy). Although most data is derived from women who underwent surgical menopause (oophorectomy), it appears that, regardless of the etiology, these women are at increased risk for osteoporosis and fractures, cognitive impairment, sexual dysfunction and cardiovascular morbidity and mortality (22).

That estrogen is critical for normal bone accrual, maintenance of bone mass and fracture prevention is indisputable. Its salutary effects have been well documented both in young hypogonadal patients (23) and during the post-menopausal period (19). On the other hand, the effects of estrogen treatment on the cognitive (24) and cardiovascular domains are controversial and seem to be age dependent.

For example, three large cohort studies encompassing more than 50,000 women (the Mayo Clinic Cohort Study of Oophorectomy and Aging, the Danish Nurse Cohort Study, and the Nurse's Health Study cohort) consistently showed increased risk of ischemic heart 
disease and cardiovascular mortality among women who underwent bilateral oophorectomy before the age of 45 (22). Women who underwent spontaneous premature menopause before the age of 40 , were also at increased risk for ischemic heart disease. In contrast to the detrimental effects found in the WHI study, estrogen therapy, particularly when instituted closely after oophorectomy provided significant cardioprotection (25). Hence, timing of treatment emerges as an important factor in determining the therapeutic effect of estrogen replacement. There is consensus that hormone replacement therapy for women with early onset hypogonadism should be continued until the mean age of physiological menopause $(\sim 51)(26)$.

\section{Estrogen preparations and route of administration}

Despite the fact that estrogen deficiency from different etiologies is not rare before the mean menopausal age, there has been no dedicated research to determine the best hormone replacement regimen for these women, and no specific guidelines exist. This is particularly striking in view of the prolonged time that these patients are exposed to treatment regimens that may have long term health consequences. Low estrogen doses recommended for postmenopausal women may not be adequate for younger women, but this has not been formally studied. The Turner Syndrome Study Group recommends doses equivalent to 2-4 mg of micronized estradiol until the age of $30 \mathrm{ys}$, subsequently reducing it to the lowest estrogen dose for the prevention of osteoporosis, although no reference is given for this recommendation (4). No similar age related dose breakdown has been indicated for early onset hypogonadotropic hypogonadism or premature ovarian insufficiency, and it is essentially unknown if dose adjustments are required throughout the adult fertile life. As already previously mentioned, treatment should resemble as much as possible age-appropriate physiology, which can be mainly achieved by the use of transdermal estradiol and natural progesterone. Although ethinyl-estradiol containing oral contraceptives are supra-physiological and have been associated with an increase incidence of venous thromboembolic events as well as with an increase risk of stroke and myocardial infarction (27), young women may find their use more convenient or age-appropriate. As no data is available as to the superiority of oral estradiol based HRT regarding venous or arterial thromboembolic events, it seems that emphasis should be given to compliance with estrogen supplementation, even if deemed less physiologic, taking into consideration the well established complications of estrogen deprivation, mainly to the skeleton.

Oral administration of estradiol leads to pharmacologic concentration of the hormone in the portal vein before it is metabolized by the liver. This first-pass effect results in increased hepatic production of several hormone binding globulins, clotting factors, lipoproteins and angiotensinogen, effects that are circumvented by transdermal administration of estrogen and have significant clinical implications. Data from post-meno- pausal women indicates that oral but not transdermal estrogen was associated with an increased odds ratio (OR $4.2,95 \% \mathrm{Cl}, 1.5$ to 11.6) for venous thromboembolism (VTE) (28). Oral estrogen administration, compared to the transdermal route caused detrimental changes in body composition, characterized by reduced lipid oxidation leading to increased fat mass, and reduced IGF-1 levels contributing to a reduction in lean body mass (29). A recent study performed in young women (19-39 ys) with premature ovarian failure showed additional beneficial effects of transdermal estrogen in that it caused significantly lower mean 24hour systolic $(-7.3 \mathrm{mmHg})$ and diastolic $(-7.4 \mathrm{~mm} \mathrm{Hg})$ blood pressures compared to oral contraceptives after 12 months (30).

\section{Progesterone preparations}

Women with an intact uterus require cyclic or continuous progestin administration to prevent endometrial hyperplasia and cancer. In the WHI study, HRT containing medroxyprogesterone acetate was associated with a higher absolute risk for adverse events, mainly related to increased cardiovascular disease and breast cancer, in comparison to the use of estrogen alone. The impact of different types of progesterone on VTE risk has been recently investigated in the case-control ESTHER study (28). There was no significant association of VTE with micronized progesterone (OR, 0.7; $95 \% \mathrm{Cl}, 0.3$ to 1.9 ) and pregnane derivatives including dydrogesterone, medrogestone, chlormadinone acetate, cyproterone acetate, and medroxyprogesterone acetate (OR, $0.9 ; 95 \% \mathrm{Cl}, 0.4$ to 2.3$)$. In contrast, norpregnane derivatives (nomegestrol acetate or promegestone) were associated with a 4-fold-increased VTE risk (OR, 3.9; $95 \% \mathrm{Cl}, 1.5$ to 10.0) (28). Based on this data, hormone replacement therapy based on transdermal estradiol and micronized progesterone seem to be the most physiologic regimen with the best safety profile, particularly in women with risk factors for VTE (31).

\section{Patients with growth hormone deficiency}

Estrogen administration reduces circulating IGF-1 levels through inhibition of growth hormone receptor signaling (32). This effect varies with the type and route of estrogen administration, being more pronounced with oral preparations that result in liver exposure to high estradiol concentrations in the portal circulation (33). Another consequence of this first pass effect is an increase in growth hormone binding protein, thus modulating growth hormone binding to its receptor. Oral ethinyl estradiol, a potent synthetic estrogen present in oral contraceptives, has a greater inhibitory effect on IGF-1 levels in comparison to natural preparations such as conjugated equine estrogen and $17 \beta$ estradiol (34). On the other hand, transdermal estradiol does not affect IGF-1 levels, unless very high doses are used. In addition to reducing IGF-1 levels through its direct ef- 
fect on growth hormone signaling, estrogen given per os increases IGFBP-1 levels thus further reducing GH metabolic actions (35). There are several clinical implications from the use of oral estrogens and in particular ethinyl estradiol, instead of transdermal estrogen, when concurrently treating $\mathrm{GH}$-deficient patients: 1) significantly higher doses of recombinant GH are needed to achieve similar or even lower IGF-1 levels, thus significantly increasing costs $(34)$; 2) the beneficial metabolic effects of GH treatment on lipid oxidation and protein synthesis are attenuated, thus compromising the expected positive effects on body composition (36).

Despite evidence of the advantages of transdermal estrogen therapy in GH-deficient/treated patients, over $80 \%$ of these young women receive oral estrogen replacement therapy, including a high percentage of oral contraceptives use (37).

\section{Conclusions}

There is accumulating evidence indicating greater safety and superior clinical outcomes when estradiol is administered using the transdermal route, spanning puberty and the fertile adult age. Transdermal estradiol should be preferentially used particularly in women with concurrent growth hormone deficiency.

\section{References}

1. Tomlinson JW, Holden N, Hills RK, et al. Association between premature mortality and hypopituitarism. Lancet 2001;357:425-431.

2. Nielsen EH, Lindholm J, Laurberg P. Excess mortality in women with pituitary disease: a meta-analysis. Clin Endocrinol 2007;67:693-697.

3. Walvoord E. Sex steroid replacement for induction of puberty in multiple pituitary hormone deficiency.PedEndocrinol Rev (PER) 2009;6, Suppl 2:298-305.

4. Bondy CA. Care of Girls and women with Turner Syndrome: a guideline of the Turner Syndrome Study Group. J Clin Endocrinol Metab 2007;92:10-25.

5. Leung KC, Johannsson G, Leong GM. Ho KKY. Estrogen regulation of growth hormone action. Endocrine Rev 2004;25:693-721.

6. Rosenfield RL, Devine N, Hunold JJ, et al. Salutary effects of combining early very low-dose systemic estradiol with growth hormone therapy in girls with Turner syndrome. J Clin Endocrinol Metab 2005; 90:6424-6430

7. Soriano-Guillen L, Coste J, Ecosse E, et al. Adult height and pubertal growth in Turner syndrome after treatment with recombinant growth hormone. J Clin Endocrinol Metab 2005;90:5197-5204.

8. Nabhan ZM, DiMeglio LA, Qi R, et al. Conjugated oral versus transdermal estrogen replacement in girls with Turner syndrome: a pilot comparative study. J Clin Endocrinol Metab 2009;94:2009-2014.

9. Paterson WF, Hollman AS, Donaldson MD.Poor uterine development in Turner syndrome with oral oestrogen therapy.Clin Endocrinol 2002;56:359-365
10. Tsilchorozidou T, Conway GS.Uterus size and ovarian morphology in women with isolated growth hormone deficiency, hypogonadotropichypogonadism and hypopituitarism.Clin Endocrinol 2004;61:567572.

11. Piipo S, Lenko H, Kainulainen P, Sipila I. The use of percutaneous estrogen gel for induction of puberty in girls with Turner syndrome. J Clin Endocrinol Metab 2004;89:3241-3247

12. Snajderova M, Mardesic T, Lebl J, et al. The uterine length in women with Turner syndrome reflects the postmenarcheal daily estrogen dose. Horm Res 2003;60:198-204.

13. Nabhan Z, Eugster EA. Hormone replacement therapy in children with hypogonadotropichypogonadism: where do we stand? Endocr Pract 2013;jun27:1-11.

14. Homburg R, Eshel A, Armar NA, et al. One hundred pregnancies after treatment with pulsatile luteinizing hormone releasing hormone to induce ovulation. BMJ 1989;298:802-813.

15. Hall R, Manski-Nankervis J, Goni N, et al. Fertility outcomes in women with hypopituitarism. Clin Endocrinol 2006;65:71-74.

16. Overton CE, Davis CJ, West C, et al. High risk pregnancies in hypopituitary women. Hum Reprod 2002;17:1464-1467.

17. Kübler K, Klingmüller D, Gembruch U, Merz WM. High-risk pregnancy management in women with hypopituitarism. J Perinatol 2009;29:89-95

18. Grodstein F, Stampfer MJ, Manson JE, et al. Postmenopausal estrogen and progestin use and the risk of cardiovascular disease. N Engl J Med 1996; 335:453-461.

19. Rossow JE, Anderson GL, Prentice RL, et al. Risks and benefits of estrogen plus progestin in healthy postmenopausal women: principal results from the Women's Health Initiative randomized controlled trial. JAMA 2002;288:321-333.

20. Sprague BL, Trentham-Dietz A, Cronin KA. A sustained secline in postmenopausal hormone use.Results from the National Health and Nutrition Examination Survey, 1999-2010.Obstet Gynecol 2012; 120:595-603.

21. Warren MP, Chua A. Appropriate use of estrogen replacement therapy in adolescents and young adults with Turner syndrome and hypopituitarism in light of the Women's Health Initiative. Growth Horm IGF Res 2006; 16:S98-S102.

22. Shuster LT, Rhodes DJ, Gostout BR, et al. Premature menopause or early menopause: long term health consequences. Maturitas 2010;65:161-166.

23. Lindsay R, Hart DM, Forrest C, Baird C. Prevention of spinal osteoporosis in oophorectomized women. Lancet 1980;2:1151-1154.

24. Rocca WA, Grossardt BR, Shuster LT. Oophorectomy, menopause, estrogen treatment, and cognitive aging: clinical evidence for a window of opportunity. Brain Res 2011; 1379:188-198.

25. Lokkegaard E, Jovanovic Z, Heitmann BL, et al. The association between early menopause and risk of ischaemic heart disease: influence of hormone therapy. Maturitas 2006; 53:226-233. 
26. Vujovic S, Brinca, M, Erel T, et al. EMAS position statement: Managing women with premature ovarian failure. Maturitas 2010; 67: 91-93.

27. Lidegaard Ø, Løkkegaard E, Jensen A, et al. Thrombotic stroke and myocardial infarction with Hormonal Contraception. N Engl J Med 2012; 366: 2257-2266.

28. Canonico M, Oger E, Plu-Bureau G, et al; for the Estrogen and Thromboembolism Risk (ESTHER) Study Group. Hormone Therapy and Venous Thromboembolism Among Postmenopausal Women. Impact of the route of estrogen Administration and progestogens: The ESTHER Study. Circulation 2007; 115:840-845

29. O'Sullivan AJ, Crampton LJ, Freund J, Ho KKY. The route of estrogen replacement therapy confers divergent effects on substrate oxidation and body composition in postmenopausal women. J Clin Invest 1998; 102:1035-1040.

30. Langrish JP, Mills NL, Bath LE, et al. Cardiovascular effects of physiological and standard sex steroid replacement regimens in premature ovarian failure. Hypertension 2009; 53:805-811.

31. Simon JA. What's new in hormone replacement therapy: focus on transdermal estradiol and micronized progesterone. Climacteric 2012:15 (suppl 1):3-10.
32. Leung KC, Johannsson G, Leong GM. Ho KKY. Estrogen regulation of Growth Hormone action. Endocrine Reviews 2004; 25:693-721.

33. Meinhardt UJ, Ho KKY. Modulation of growth hormone action by sex steroids. Clin Endocrinol 2006; 65:413-422.

34. Phelan N, Conway SH, Liahana S, Conway GS. Quantification of the adverse effect of ethinylestradiol containing oral contraceptive pills when used in conjunction with growth hormone replacement in routine practice. Clin Endocrinol 2012; 76:729-733.

35. Isotton AL, Wender MCO, Casagrande A, et al. Effects of oral and trandermal estrogen on IGF-1, IGFBP-3, IGFBP-1, serum lipids and glucose in patients with hypopituitarism during $\mathrm{GH}$ treatment: a randomized study. Eur J Endocrinol 2012; 166:207213.

36. Wolthers T, Hoffman DM, Nugent AG, et al. Oral estrogen antagonizes the metabolic actions of growth hormone in growth hormone-deficient women. Am J Physiol Endocrinol Metab 2001; 281: E1191E1196.

37. Mah PM, Webster J, Jonsson P, et al. Estrogen replacement in women of fertile years with hypopituitarism. J Clin Endocrinol Metab 2005; 90:5964-5969. 